\title{
Dynamic Workflow Modeling and Verification
}

\author{
Jiacun Wang and Daniela Rosca \\ Department of Software Engineering, \\ Monmouth University, \\ West Long Branch, NJ 07762, USA \\ \{jwang, drosca\} @monmouth. edu
}

\begin{abstract}
The dynamic nature of incident command systems and their requirement for high flexibility raise a challenge to the research and implementation of workflows. The significance of applying formal approaches to the modeling and analysis of workflows has been well recognized and several such approaches have been proposed. However, these approaches require users to master considerable knowledge of the particular formalisms, which impacts their application on a larger scale. To address these challenges, we developed an intuitive, yet formal approach to workflow modeling, enactment and validation. In this paper, we further develop a set of theorems to support dynamic modeling, modification and on-the-fly verification of the workflows. A prototype has been implemented to demonstrate the feasibility of the theoretical approach.
\end{abstract}

\section{Introduction}

The business environment today is undergoing rapid and constant changes. The way companies do business, including the business processes and their underlying business rules, ought to adapt to these changes rapidly with minimum interruption of the ongoing operations [9, 13]. This flexibility becomes of a paramount importance in applications such as incident command systems (ICS) that support the allocation of people, resources and services in the event of a major natural or terrorist incident. These systems have to deal with a predominantly volunteer-based workforce and frequent changes in the course of execution of their workflows, dictated by unplanned incoming events [19].

Dealing with these issues generates many challenges for a workflow management system (WFMS). The necessity of making many ad-hoc changes calls for an on-thefly verification of the correctness of the modified workflow. This cannot be achieved without an underlying formal approach of the workflow, which does not leave any scope for ambiguity and sets the ground for analysis. Yet, since our main users will be volunteers from various backgrounds, with little computer experience, we need to provide an approach with highly intuitive features for the description and modification of the workflows.

A number of formal modeling techniques have been proposed in the past decades for modeling processes and business rules [5, 15, 1, 3, 9]. Petri nets are one of the most widely used approaches because of its formal semantics as well as graphical nature [2]. However, they model the loops implicitly, making the decision of whether 
a cycle is desired in the workflow, or it is a deadlock, a NP-hard problem. Other than Petri Nets, techniques such as state charts have also been proposed for modeling WFMS [12]. Although state charts can model the behavior of workflows, they have to be supplemented with logical specification for supporting analysis. Singh et al [16] use event algebra to model the inter-task dependencies and temporal logic. Attia et al [7] have used computational tree logic to model workflows.

As indicated in [3], it is desirable that a business process model can be understood by the stakeholders as straightforwardly as possible. Unfortunately, a common major drawback of all the above formal approaches is that only users who have the expertise in these particular formal methods can build their workflows and dynamically change them. For example, in order to add a new task to a Petri-net based workflow, one must manipulate the model in terms of transitions, places, arcs and tokens, which can be done correctly and efficiently only by a person with a good understanding of Petri-nets. This significantly affects the application of these approaches on a large scale. To address this issue, we introduced a new Workflows Intuitive Formal Approach (WIFA) for the modeling and analysis of workflows, which, in addition to the abilities of supporting workflow validation and enactment, possesses the distinguishing feature of allowing users who are not proficient in formal methods to build up and dynamically modify the workflows that address their business needs [19]. In this paper, we further develop a set of theorems to guide the dynamic, well-formed workflow modeling, modification and verification. Since WIFA is a result of the desire of modeling the ICS, it does not make the distinction between workflow schemas and workflow instances. There is only one person executing a dedicated workflow at a particular time in an ICS. However, after undertaking the modeling of other applications, we have noticed the need of introducing this distinction. The discussion of workflow instance migration to new schemas during the workflow execution is outside the scope of this paper.

Although WIFA was designed with a high degree of usability in mind, it has not sacrificed expressive power. As such, WIFA is able to model sequential and concurrent execution of tasks, conflict resolution, synchronization, mutual exclusion and loops. MILANO [6], another tool that claims "simplicity" of use, has sacrificed some expressive power, such as the representation of loops, for the flexibility during enactment. The same deficiency can be noticed in WASA [20]. WIDE [8] proposed a complete and minimal set of primitives that allow the correct transformation of an old workflow schema to a new one. Based on that minimal set, other change primitives can be derived, for both modifying workflow schemas and migrating instances to new schemas. TRAM [11], uses a versioning approach for the modification of workflow schemas. They use a principle similar to WASA's for migrating workflow instances to new schemas, e.g. verifying whether the instance can continue from its current state according to the new schema. In [5], the author proposes an approach where schema modifications should not be migrated to instances that are executed on "change regions". Currently, WIFA does not handle data flow control, as in WASA, Flow Nets [10], ADEPT [14], and other systems. This represents a dimension that needs to be added to our work.

The paper is organized as follows: Section 2 briefly introduces the new workflow formalism WIFA and its state transition rules. A detailed presentation can be found in [19]. Section 3 presents the definition of well-formed workflows and a set of theorems which help build well-formed workflows and dynamically validate workflows 
after various types of modifications. In Section 4, an example is used to illustrate the use of these theorems. Section 5 presents a brief description of the prototype that supports the WIFA approach, as well as a discussion of the findings of a usability study conducted to assess WIFA's intuitiveness. Finally, Section 6 presents conclusions and ideas for the continuation of this work.

\section{The WIFA Workflow Model}

In general, a workflow consists of processes and activities, which are represented by well-defined tasks. Two tasks are said to have precedence constraints if they are constrained to execute in some order. As a convention, we use a partial-order relation <, called a precedence relation over the set of tasks, to specify the precedence constraints among tasks. A classic way to represent the precedence constraints among tasks in a set $T$ is by a directed graph $G=(T,<)$, in which each vertex represents a task in $T$, and there is a directed edge from vertex $T_{i}$ to vertex $T_{j}$ if $T_{i}$ is an immediate predecessor of $T_{j}$. The graph is called a precedence graph.

The preset of a task $T_{k}$ is the set of all tasks that are immediate predecessors of the task, denoted by $* T_{k}$; the postset of $T_{k}$ is the set of all tasks that are immediate successors of the tasks, denoted by $T_{k}{ }^{*}$. If $\left|T_{k} *\right| \geq 1$, then the execution of $T_{k}$ might trigger multiple tasks. Suppose $\left\{T_{i}, T_{j}\right\} \subseteq T_{k}{ }^{*}$. There are two possibilities: (1) $T_{i}$ and $T_{j}$ can be executed simultaneously, and (2) only one of them can be executed, and the execution of one will disable the other, due to the conflict between them. We denote the former case by $c_{i j}=c_{j i}=0$, and the latter case by $c_{i j}=c_{j i}=1$.

If $\left|* T_{k}\right| \geq 1$, then based on the aforementioned classic precedence model, the execution of $T_{k}$ won't start until all of its immediate predecessors are executed. This precedence constraint is called AND precedence constraint. An extension to this classic precedence model is to allow a task to be executed when some of its immediate predecessors are executed. This loosens the precedence constraints to some extent, and the loosened precedence constraint is called $O R$ precedence constraint. Obviously, the $O R$ precedence model provides more flexibility than the classic $A N D$ precedence model in describing the dependencies among tasks. In this paper, the $O R$ precedence model is adopted. The $A N D$ precedence model can be viewed as a special case of the $O R$ precedence model.

\subsection{WIFA Workflow Definition}

In WIFA, a workflow is defined as a 5-tuple: $W F=\left(T, P, C, A, S_{0}\right)$, where

1) $T=\left\{T_{1}, T_{2}, \ldots, T_{m}\right\}$ is a set of tasks, $m \geq 1$.

2) $P=\left(p_{i j}\right)_{m \times m}$ is the precedence matrix of the task set. If $T_{i}$ is the immediate predecessor of $T_{j}$, then $p_{i j}=1$; otherwise, $p_{i j}=0$.

3) $C=\left(c_{i j}\right)_{m \times m}$ is the conflict matrix of the task set. $c_{i j} \in\{0,1\}$ for $i=1,2, \ldots m$ and $j$ $=1,2, \ldots m$.

4) $A=\left(A\left(T_{1}\right), A\left(T_{2}\right), \ldots, A\left(T_{m}\right)\right)$ defines pre-condition set for each task. $\forall T_{k} \in T$, $A\left(T_{k}\right): * T_{k} \rightarrow 2^{* T_{k}}$. Let set $A^{\prime} \in A\left(T_{k}\right)$. Then $T_{i} \in A^{\prime}$ implies $p_{i k}=1$.

5) $S_{0} \in\{0,1,2,3\}^{m}$ is the initial state of the workflow. 
A state of the $W F$ is denoted by $S=\left(S\left(T_{1}\right), S\left(T_{2}\right), \ldots, S\left(T_{m}\right)\right)$, where $S\left(T_{i}\right) \in\{0,1$, $2,3\} . S\left(T_{i}\right)=0$ means $T_{i}$ is not executable at state $S$ and not executed previously; $S\left(T_{i}\right)$ $=1$ means $T_{i}$ is executable at state $S$ and not executed previously; $S\left(T_{i}\right)=2$ means $T_{i}$ is not executable at state $S$ and executed previously; and $S\left(T_{i}\right)=3$ means $T_{i}$ is executable at state $S$ and executed previously.

By the above definition of state values, at any state, only those tasks whose values are either 1 or 3 can be selected for execution. Suppose task $T_{i}$ at state $S_{a}$ is selected for execution, and the new state resulted from the execution of $T_{i}$ is $S_{b}$, then the execution of $T_{i}$ is denoted by $S_{a}\left(T_{i}\right) S_{b}$.

At the initial state $S_{0}$, for any task $T_{i} \in T$, if there is no $T_{j}$ such that $p_{j i}=1$, then $S_{0}\left(T_{i}\right)=1$; otherwise $S_{0}\left(T_{i}\right)=0$.

Note that tasks that have no predecessor do not need to wait for any other task to execute first. In other words, these tasks are executable immediately. We assume that there are always such tasks in a workflow. They are the initial triggers or "starting" tasks of workflows.

Fig. 1 shows a workflow model with seven tasks, $T=\left\{T_{1}, T_{2}, \ldots T_{7}\right\}$, in which $T_{1}$ is the starting task of the workflow. The execution of $T_{1}$ triggers both $T_{2}$ and $T_{3}$, which do not conflict with each other, i.e., $c_{23}=c_{32}=0 . T_{2}$ can be triggered by either $T_{1}$ or $T_{6}$, i.e., $A\left(T_{2}\right)=\left\{\left\{T_{1}\right\},\left\{T_{6}\right\}\right\}$. The execution of $T_{5}$ triggers both $T_{6}$ and $T_{7}$, which conflict with each other, i.e., $c_{67}=c_{76}=0 . T_{7}$ is executable only if both $T_{3}$ and $T_{5}$ are executed, i.e., $A\left(T_{7}\right)=\left\{\left\{T_{3}\right\},\left\{T_{5}\right\}\right\}$. The initial state is $S_{0}=(1,0,0,0,0,0,0)$. After the execution of $T_{1}$, the new state will be $S_{1}=(2,1,1,0,0,0,0)$. If in the next step we select $T_{2}$ for execution, the new state will be $S_{2}=(2,2,1,1,0,0,0)$.

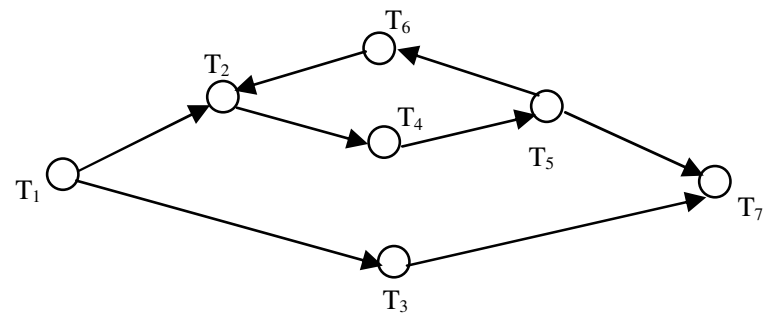

Fig. 1. A seven-task workflow

\subsection{State Transition Rules}

The dynamics of a WIFA workflow can be captured by state transitions. The state transitions are guided by the following rules:

If $S_{a}\left(T_{i}\right) S_{b}$, then $\forall T_{j} \in T$,

1) If $T_{j}=T_{i}$ then $S_{b}\left(T_{j}\right)=2$;

2) If $T_{j} \neq T_{i}$ then the state value of $T_{j}$ at new state $S_{b}$ depends on its state value at state $S_{a}$. We consider four cases:

Case $A-S_{a}\left(T_{j}\right)=0$ :

If $p_{i j}=1$ and $\exists A \in A\left(T_{j}\right)$ such that $S_{b}\left(T_{k}\right)=2$ for any $T_{k} \in A$, then $S_{b}\left(T_{j}\right)=1$; otherwise $S_{b}\left(T_{j}\right)=0$. 


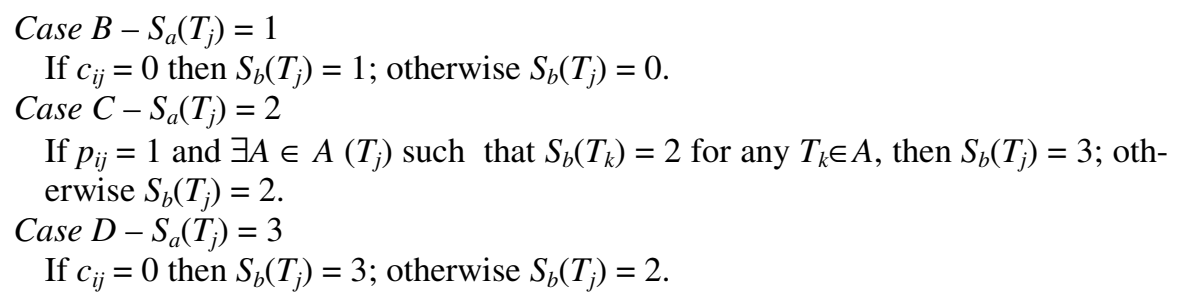

According to the above state transition rules, for example, a task's state value at a given state other than the initial state is 0 iff one of the following is true:

1) Its state value is 0 in the previous state and it is not a successor of the task which is just executed.

2) Its state value is 0 in the previous state, and it is the successor of the task which is just executed, but for each of its precondition sets there is at least one task that is not executed.

3) Its state value is 1 in the previous state but it conflicts with the task which is just executed.

Note that a state value can increment from 0 to 1 , from 1 to 2 or from 2 to 3 ; it can also decrement from 1 to 0 or from 3 to 2 . But it cannot decrement from 2 to 1 .

\section{Well-Formed Workflow Definitions}

In this section, we introduce well-formed workflows which have no dangling tasks and are guaranteed to finish. We particularly discuss confusion-free workflows, which are a class of well-formed workflows and have some distinguishing properties. We demonstrate how to build confusion-free workflows, and how to ensure a workflow remains confusion-free when it is changed.

\subsection{Well-Formed Workflow Definitions}

Definition 1 (execution path). An execution path is a sequence of tasks that are executable starting from a given state. Denote all possible execution paths starting from a state $S$ by $\Sigma(S)$.

Definition 2 (reachable set). A state $S_{k}$ of a workflow is reachable from the initial state $S_{0}$ if and only if there exists $\sigma \in \Sigma\left(S_{0}\right)$ such that the execution of $\sigma$ leads the workflow to state $S_{k}$. denoted by $S_{0}(\sigma) S_{k}$. The set of all reachable states, including the initial state, is called the reachable set of a workflow, denoted by $R$.

Definition 3 (well-formed workflow). A workflow is well-formed if and only if the following two behavior conditions are met:

1) $\forall T_{i} \in T, \exists S \in R$ such that $S\left(T_{i}\right)=1$. (i.e. there is no dangling task.)

2) $\forall S_{i} \in \mathcal{R}, \exists \sigma \in \Sigma\left(S_{i}\right)$ and $S_{e} \in \mathcal{R}$, where $S_{e}\left(T_{i}\right) \in\{0,2\}$ for $\forall T_{i} \in T$, such that $S_{i}(\sigma) S_{e}$. (i.e., given any reachable state, there is always a path leading the workflow to finish.) 
The example workflow given in Section 2.1 is well-formed, because based on Fig. 1, every task in this workflow is executable, and from each state there is an execution path leading the workflow to one of the two ending states. In general, the validation of a workflow being well-formed requires the reachability analysis of the workflow. Below we introduce confusion-free workflows, which are a class of wellformed workflows with some restrictions imposed on their structure.

Definition 4 (confusion-free workflow). A well-formed workflow is confusion-free if and only if the following two structural conditions are met:

1) $\forall T_{k} \in T$ with $\left|T_{k} *\right| \geq 3$, if $\exists T_{i}, T_{j} \in T_{k}{ }^{*}$ such that $c_{i j}=1$ (or $c_{i j}=0$ ), then for $\forall T_{a}$, $T_{b} \in T_{k} * c_{a b}=1$ (or $c_{a b}=0$ ) (i.e., either all tasks triggered by the task are in conflict, or no pair of them are in conflict. In the former case, the task is called an AND-out task; in the latter case, it is called a XOR-out task.)

2) $\forall T_{k} \in T$ with $* T_{k}=\left\{T_{k 1}, T_{k 2}, \ldots, T_{k n}\right\}, n \geq 2$, either

$$
A\left(T_{k}\right)=\left\{\left\{T_{k 1}, T_{k 2}, \ldots, T_{k n}\right\}\right\},
$$

or

$$
A\left(T_{k}\right)=\left\{\left\{T_{k 1}\right\},\left\{T_{k 2}\right\}, \ldots,\left\{T_{k n}\right\}\right\}
$$

(i.e., $T_{k}$ becomes executable either when all of its predecessor tasks are executed, or when any one of them is executed. In the former case, the task is called an AND-in task; in the latter case, it is called a XOR-in task.)

Based on this definition, the example workflow of Fig. 1 is confusion-free. As will be described next, it is easy to construct and validate a confusion-free workflow.

From the perspective of triggering conditions and relations among triggered tasks, we can classify the tasks in a confusion-free well-formed workflow into four types: AND-in-AND-out, AND-in-XOR-out, XOR-in-AND-out and XOR-in-XOR-out.

Without loss of generality, a task with only one or no immediate predecessor is treated as an "AND-in" task, and a task with only one or no immediate successor treated as an "AND-out" task. We denote by $T_{A P}$ the set of all AND-in-AND-out tasks, $T_{A C}$ all AND-in-XOR-out tasks, $T_{O P}$ all XOR-in-AND-out tasks, and $T_{O C}$ all XOR-in-XOR-out tasks. For example, for the workflow of Fig. 1, $T_{A P}=\left\{T_{1}, T_{3}, T_{4}\right.$, $\left.T_{6}, T_{7}\right\}, T_{A C}=\left\{T_{5}\right\}, T_{O P}=\left\{T_{2}\right\}$, and $T_{O C}=\varnothing$.

Since the definition of well-formed workflows has a concern over infinite task loop, we formally define a task loop as follows:

Definition 5 (loop). Tasks $T_{1}, T_{2}, \ldots, T_{s}$ forms a loop $L$ iff $p_{s, 1}=p_{i, i+1}=1$ for $i=1$, $2, \ldots, s$-1. Let $T(L)=\left\{T_{1}, T_{2}, \ldots, T_{k}\right\}$. A task $T_{k} \in T \backslash T(L)$ is said to be an entry of $L$ iff $p_{k j}=1$ for some $T_{j} \in T(L)$. A task $T_{k} \in T \backslash T(L)$ is said to be an exit of $L$ iff $p_{j k}=1$ for some $T_{j} \in T(L)$.

In the workflow of Fig. 1, for example, there is a loop $L$ where $T(L)=\left\{T_{2}, T_{4}, T_{5}, T_{6}\right\}$. For this loop, $T_{1}$ is the only entry task, while $T_{7}$ the only exit task. It is obvious that if a loop has no entry task, then all tasks in the loop are dangling tasks; if a loop has no exit task, then the loop is an infinite loop, and the workflow will never be finished. In the rest of the paper, a loop with at least one entry task and one exit task is called a healthy loop. Otherwise, the loop is called an unhealthy loop. 
Discussion: WIFA engine in general allows us to build any control pattern of workflow constructs as mentioned in [4]. However, a well-formed confusion-free WIFA model can only be composed of the five simple control patterns, namely sequence, parallel split, synchronization, exclusive choice and simple merge.

\subsection{Build a Well-Formed Workflow}

Lemma 1: Given a workflow $W F_{A}=\left(T, P, C, A, S_{0}\right)$ with $T_{k} \in T$. As shown in Fig. 2, $W F_{B}=\left(T^{\prime}, P^{\prime}, C^{\prime}, A^{\prime}, S^{\prime}{ }_{0}\right)$ is obtained by replacing $T_{k}$ with $T_{k 1}$ and $T_{k 2}$, such that

1) $* T_{k 1}=* T_{k}, T_{k 2} *=T_{k} *, T_{k 1} *=\left\{T_{k 2}\right\}$ and $* T_{k 2}=\left\{T_{k 1}\right\}$,

2) $A^{\prime}\left(T_{k l}\right)=A\left(T_{k}\right)$, i.e., $T_{k l}$ has the same pre-condition set in $W F_{B}$ as that of $T_{k}$ in $W F_{A}$;

3) $C^{\prime}\left(T_{i}, T_{j}\right)=C\left(T_{i}, T_{j}\right)$ for $\forall T_{i}, T_{j} \in T_{k}{ }^{*}$, i.e, the conflict property among all $T_{k}$ 's immediate successors remain unchanged in $W F_{B}$.

Then $W F_{B}$ is confusion-free well-formed iff $W F_{A}$ is confusion-free well-formed.

\section{Proof}

Necessity: Assume $W F_{A}$ is well-formed. Then there exists an execution path which results in reachable state sequence $S_{0} S_{1} S_{2} \ldots S_{a-1} S_{a}$ with $W F_{A}$ such that:

$$
\begin{aligned}
& S_{i}\left(T_{k}\right)=0, i=0,1, \ldots a-1 ; \\
& S_{a}\left(T_{k}\right)=1 .
\end{aligned}
$$
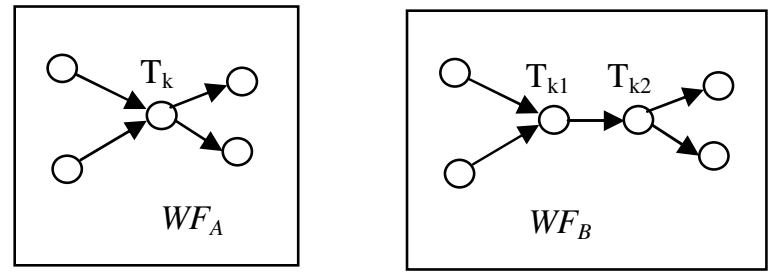

Fig. 2. $W F_{A}$ and $W F_{B}$ in Lemma 1

Based on the definition of $W F_{B}$, we know there is a corresponding state sequence $S_{0}{ }_{0} S_{1} S^{\prime}{ }_{2} \ldots S^{\prime}{ }_{a-1} S_{a}{ }_{a}$ with $W F_{B}$ such that:

$$
\begin{aligned}
& S_{i}^{\prime}=S_{i} \cup\left\{S_{i}\left(T_{k 1}\right)\right\} \cup\left\{S_{i}\left(T_{k 2}\right)\right\} \backslash\left\{S_{i}\left(T_{k}\right)\right\}, i=0,1, \ldots a ; \\
& S_{i}^{\prime}\left(T_{k 1}\right)=S^{\prime}{ }_{i}\left(T_{k 2}\right)=0, i=0,1, \ldots a-1 ; \\
& S^{\prime}{ }_{a}\left(T_{k 1}\right)=1, S^{\prime}{ }_{a}\left(T_{k 2}\right)=0 .
\end{aligned}
$$

After the execution of $T_{k 1}$ at $S_{a}^{\prime}$, task $T_{k 2}$ becomes executable. Then based on conditions 1) and 3), it is obvious that the behavior of $W F_{B}$ after the execution of task $T_{k 2}$ will be the same as that of $W F_{A}$ after the execution of task $T_{k}$. So $W F_{B}$ is also well-formed.

Sufficiency: Assume $W F_{B}$ is well-formed. Then there exists an execution path which results in reachable state sequence $S_{0} S_{1}{ }_{1} S_{2}{ }_{2} \ldots S_{a-1} S_{a} S^{\prime}{ }_{a+1}$ with $W F_{B}$ such that: 


$$
\begin{aligned}
& S_{i}^{\prime}\left(T_{k 1}\right)=S^{\prime}{ }_{i}\left(T_{k 2}\right)=0, i=0,1, \ldots a-1 ; \\
& S_{a}{ }_{a}\left(T_{k 1}\right)=1, S^{\prime}{ }_{a}\left(T_{k 2}\right)=0 . \\
& S^{{ }_{a+l}}\left(T_{k 1}\right)=2, S^{\prime}{ }_{a+l}\left(T_{k 2}\right)=1 .
\end{aligned}
$$

Based on the relationship between $W F_{A}$ of $W F_{B}$, there exists a corresponding state sequence $S_{0} S_{1} S_{2} \ldots S_{a-1} S_{a}$ with $W F_{A}$ such that:

$$
\begin{aligned}
& S_{i}=S_{i} \cup\left\{S_{i}\left(T_{k}\right)\right\} \backslash\left\{S_{i}\left(T_{k 1}\right)\right\} \backslash\left\{S_{i}\left(T_{k 2}\right)\right\}, i=0,1, \ldots a ; \\
& S_{i}\left(T_{k}\right)=0, i=0,1, \ldots a-1 ; \\
& S_{a}\left(T_{k}\right)=1 .
\end{aligned}
$$

According to conditions 1) and 3), the behavior of $W F_{A}$ after state $S_{a}$ will be the same as that of $W F_{B}$ after $S_{a+1}$. So $W F_{A}$ is also well-formed.

The lemma is proved.

Lemma 2: Let $W F_{A}=\left(T, P, C, A, S_{0}\right)$ be a well-formed confusion-free workflow with $T_{k 1}, T_{k 2} \in T, T_{k 1}{ }^{*}={ }^{*} T_{k 2}=\varnothing$, and $T_{k 2}$ is not a predecessor of $T_{k 1}$. As shown in Fig. 3, $W F_{B}=\left(T^{\prime}, P^{\prime}, C^{\prime}, A^{\prime}, S^{\prime}{ }_{0}\right)$ is obtained by introducing precedence constraint between $T_{k 1}$ and $T_{k 2}$ such that $T_{k 1}$ is an immediate predecessor of $T_{k 2}$. Then $W F_{B}$ is also wellformed and confusion-free.
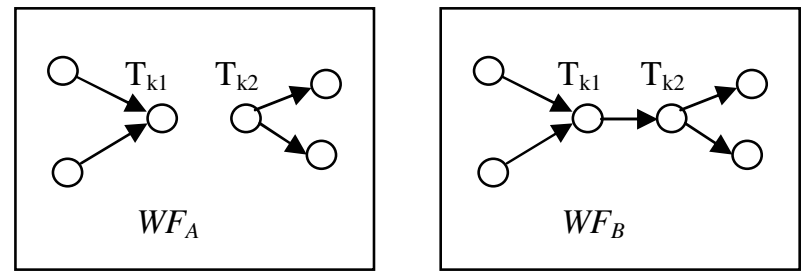

Fig. 3. $W F_{A}$ and $W F_{B}$ in Lemma 2

Proof: Since $W F_{A}$ is well-formed, so $T_{k 1}$ is executable. Also because $T_{k 2}$ is not a predecessor of $T_{k l}$, there exists an execution path which results in reachable state sequence $S_{0} S_{1} S_{2} \ldots S_{a-1} S_{a}$ with $W F_{A}$ such that:

$$
\begin{aligned}
& S_{i}\left(T_{k l}\right)=0, i=0,1, \ldots a-1 ; \\
& S_{a}\left(T_{k l}\right)=1 ; \\
& S_{i}\left(T_{k 2}\right)=0, i=0,1, \ldots a .
\end{aligned}
$$

Based on the definition of $W F_{B}$, for each such a sequence with $W F_{A}$, we know there is a corresponding state sequence $S_{0}{ }_{0}{ }^{\prime}{ }_{1} S_{2}{ }_{2} \ldots S^{\prime}{ }_{a-1} S^{\prime}{ }_{a}$ with $W F_{B}$ such that:

$$
\begin{aligned}
& S_{i}^{\prime}\left(T_{k 1}\right)=0, i=0,1, \ldots a-1 ; \\
& S^{\prime}{ }_{a}\left(T_{k 1}\right)=1 ; \\
& S_{i}^{\prime}\left(T_{k 2}\right)=0, i=0,1, \ldots a .
\end{aligned}
$$

After the execution of $T_{k 1}$ at $S_{a}$, task $T_{k 2}$ becomes executable, and all $T_{k 2}$ 's successor tasks in $W F_{B}$ are executable as they are in $W F_{A}$. Because $T_{k 2}$ is not a predecessor of 
$T_{k l}$, no new loop is introduced, so there won't be any infinite loop in $W F_{B}$, thus $W F_{B}$ can always finish. This means $W F_{B}$ is well-formed and confusion free.

The lemma is proved.

Theorem 1: Given a confusion-free well-formed workflow $W F=\left(T, P, C, A, S_{0}\right)$, by adding a new task $T_{k}$ to it, the obtained new workflow is denoted by $W F^{\prime}=\left(T^{\prime}, P^{\prime}\right.$, $C^{\prime}, A^{\prime}, S_{0}$ '). Then $W F^{\prime}$ is also a confusion-free workflow if it matches one of the following cases:

1) $* T_{k}=T_{k}{ }^{*}=\varnothing$, i.e., $p^{\prime}{ }_{k i}=p^{\prime}{ }_{i k}=0$ for all $T_{i} \in T^{\prime} \backslash\left\{T_{k}\right\}$.

2) $* T_{k}=\varnothing, T_{k}^{*} \neq \varnothing$, and $\forall T_{i} \in T_{k}^{*}$, if $T_{k}$ is an AND-in task in $W F$, then $T_{i}$ is also an AND-in task in $W F^{\prime}$; If $T_{i}$ is an XOR-in task in $W F$, then $T_{i}$ is also an XOR-in task in $W F^{\prime}$.

3) $* T_{k} \neq \varnothing, T_{k}^{*}=\varnothing$. If $T_{k}$ is an all-in task in $W F$, then there exists a $S_{a} \in R(W F)$ such that $S_{a}\left(T_{i}\right)=2$ for all $T_{i} \in * T_{k}$; If $T_{k}$ is an or-in task in $W F$, then there exists a $S_{a} \in$ $\mathcal{R}(W F)$ such that $S_{a}\left(T_{i}\right)=2$ for some $T_{i} \in{ }^{*} T_{k}$. In addition, $\exists T_{i} \in{ }^{*} T_{k}$, if $T_{i}$ triggers two or more conflicting tasks, then $T_{k}$ conflicts with each of these tasks, otherwise, $c_{k j}=0$ for any $T_{j} \in T_{i}^{*}$.

4) $* T_{k} \neq \varnothing, T_{k}^{*} \neq \varnothing$, with all other conditions appear in 2) and 3). Besides, $\forall T_{i} \in$ $T_{k}{ }^{*}$, if $T_{i}$ is also a predecessor of $T_{k}$ (i.e., $T_{k}$ introduces a loop), then $T_{i}$ can only be an XOR-in task, and the loop is a healthy loop.

\section{Proof}

Case 1): $T_{k}$ is an isolated task. Based on Definition 3, $T_{k}$ will not be in any other task's pre-condition set, so it has no impact to the original workflow $W F$, and the two structural conditions of confusion-free workflows are all met in $W F^{\prime}$. Because $T_{k}$ has no predecessors, so it is executable in $S_{0}{ }_{0}$. Since $W F$ is well-formed, there must be an ending state $S_{q} \in \mathbb{R}(W F)$, then state $S^{\prime}{ }_{q}=S_{q} \cup\left\{S\left(T_{k}\right)=2\right\}$ is an ending state of $W F$ '. Therefore, $W F$ ' is confusion-free.

Case 2): In this case, $T_{k}$ has no predecessors, so it is executable in $S_{0}{ }_{0}$. We need to make sure that all tasks that are successors to $T_{k}$ are still executable after adding in $T_{k}$. As shown in Fig. 7(b), $\forall T_{i} \in T_{k}{ }^{*}$, if $T_{i}$ is an and-in task in $W F$ ', then that $W F$ is confusion-free indicates that there is a state $S_{a}$ in $W F$ such that all tasks in $* T_{i}$ have state value of 2. Because $T_{k}$ is unconditionally executable, so there must be a corresponding state $S_{a}{ }^{\prime}$ in $W F^{\prime}$ such that $S_{a}{ }^{\prime}=S_{a} \cup\left\{S_{a}{ }^{\prime}\left(T_{i}\right)=2\right\}$. Thus $T_{i}$ is still executable in $W F^{\prime}$. On the other hand, if $T_{i}$ is an or-in task in $W F$, then the execution of any task in ${ }^{*} T_{i}$ in $W F$ ' can still trigger $T_{i}$ as it does in $W F$, and $T_{k}$ is just an additional task that triggers $T_{i}$. Thus $T_{i}$ is still executable in $W F^{\prime}$.

Now consider the second condition of being well-formed workflows. Since $W F$ is well-formed, $\forall S_{i} \in \mathbb{R}(W F), \exists \sigma \in \Sigma\left(S_{i}\right)$ and an ending state $S_{e} \in \mathbb{R}(W F)$, such that $S_{i}(\sigma) S_{e}$. If $T_{i}$ has no occurrence in $\sigma$, then for $W F$ ' we have

$$
S_{i} \cup\left\{S\left(T_{k}\right)=0\right\}\left(\sigma T_{k}\right) S_{e} \bigcup\left\{S\left(T_{k}\right)=2\right\},
$$

where $S_{i} \cup\left\{S\left(T_{k}\right)=0\right\}$ is the state in $W F^{\prime}$ which is the $S_{i}$ extended with the state of $T_{k}$, $S_{e} \cup\left\{S\left(T_{k}\right)=2\right\}$ the state in $W F^{\prime}$ ' which is the $S_{e}$ extended with the state of $T_{k}$, and $\sigma T_{k}$ the execution path of $W F$ '. If $T_{i}$ has one occurrence in $\sigma$, let $\sigma$ be $\sigma_{1} T_{i} \sigma_{2}$. Then for $W F$ ' we have 


$$
S_{i} \cup\left\{S\left(T_{k}\right)=0\right\}\left(\sigma_{1} T_{k} T_{i} \sigma_{2}\right) S_{e} \bigcup\left\{S\left(T_{k}\right)=2\right\},
$$

If $T_{i}$ has multiple occurrences in $\sigma$, we can use the same way to construct the execution path for $W F$ ' which leads it to finish.

Case 3): In this case, $T_{k}$ has no successors. The other conditions already guarantee that task $T_{k}$ is executable, and the two structural conditions of confusion-free workflows are also met. We only need to prove that the introduction of $T_{k}$ won't cause other tasks to become non-executable. It is easy to understand that the state transition behavior of $W F^{\prime}$ from any state $S^{\prime}$ in which $S^{\prime}\left(T_{k}\right)=0$ is not affected due to the introduction of $T_{k}$. Suppose that at state $S_{a}$ ' we have $S_{a}{ }^{\prime}\left(T_{k}\right)=1$ and $T_{k}$ is triggered by $T_{i}\left(T_{i}\right.$ $\left.\in{ }^{*} T_{k}\right)$. If all tasks triggered by $T_{i}$ are able to execute in parallel with $T_{k}\left(c_{k j}=0\right.$ for any $\left.T_{i} \in T_{i}^{*}\right)$, then $T_{k}$ has no impact to the execution of other triggered tasks. The other possibility is that $T_{k}$ is in conflict with any other task triggered by $T_{i}$. In this case, if $T_{k}$ is not chosen for execution, the state transition behavior from $S^{\prime}$ will be just like the case in state $S=S^{\prime} \backslash\left\{S^{\prime}\left(T_{k}\right)=1\right\}$ of $W F$. All these suggests that $W F^{\prime}$ is also a confusion-free workflow.

Case 4): This case can be viewed as a combination of Case 2 and Case 3. If $T_{k}$ doesn't introduce a loop to the workflow, then we can add $T_{k 1}$ and $T_{k 2}$ to $W F$ first, where $T_{k l}$ satisfies condition 3) and $T_{k 2}$ satisfies condition 2). The obtained workflow is like the $W F_{A}$ shown in Fig.5 and it is well-formed and confusion free as we just proved in Case 2 and Case 3. Then by connecting $T_{k 1}$ and $T_{k 2}$ and applying Lemma 2, we know obtained workflow, which is illustrated as $W F_{B}$ in Fig. 5, is well-formed and confusion free. Merging $T_{k 1}$ and $T_{k 2}$ and applying Lemma 1, we conclude that $W F$, which is illustrated as $W F_{A}$ in Fig. 4, is also well-formed and confusion free.

In case $T_{k}$ introduces a loop to the workflow, since we already restrict that $T_{i}$ be an or-in task, $T_{i}$ can be triggered as it is without $T_{k}$ in place. Adding $T_{k}$ simply introduces one more trigger to $T_{i}$. So the loop does not cause any task un-executable. Moreover, because the loop is healthy, the workflow can finish.

The theorem is proved.

Theorem 1 can serve as a rule in building a well-formed and confusion-free workflow. At the beginning, the task set is empty. When the first task is introduced, the workflow is well-formed, because this single task has no predecessors and successors and it is executable. Then we add a second task. This second task can either be an isolated one (Case 1 of Theorem 1), or be a successor of the first task (Case 2 of Theorem 1), or be a predecessor of the first task (Case 3 of Theorem 1), or even be both a predecessor and successor to the first task (Case 4 of Theorem 1). Since the first task is the only possible successor or predecessor to the second task, the new workflow (with these two tasks) is still confusion-free. When we continue to introduce more tasks to the workflow, as long as we make sure each new task is added in such a way that it satisfies the conditions defined in one of the four cases, then the new workflow is guaranteed to be confusion-free.

\subsection{Modify a Well-Formed Workflow}

Modifying a workflow can be conducted in three ways: adding new tasks to the workflow, deleting tasks from the workflow, and changing business rules defined on the workflow by adding or deleting precedence arcs between tasks. Theorem 1 considered 
the case of adding new tasks to a workflow. Now we consider the last two types of modifications. We first discuss deleting a precedence arc from a workflow, then deleting a task, and then adding precedence arcs.

Theorem 2: Let $W F=\left(T, P, C, A, S_{0}\right)$ be a confusion-free well-formed workflow with $T_{i}, T_{j} \in T$ and $p_{i j}=1$. WF' is obtained by deleting the precedence arc between $T_{i}$ and $T_{j}$, i.e. setting $p_{i j}$ to 0 . Then $W F$ ' is confusion-free well-formed iff the deletion does not introduce any unhealthy loop.

Proof: Because $W F$ is confusion-free well-formed, so $T_{j}$ is executable in $W F$. Also because in $W F$ ' the precondition of $T_{j}$ is loosened compared with it is in $W F$, so $T_{j}$ is executable in $W F$ ' as well. This means the executability of $T_{j}$ and all its successors are not affected by deleting removing the precedence relation between $T_{i}$ and $T_{j}$. Also because the precedence arc deletion does not introduce any unhealthy loop, the workflow is guaranteed to finish. Therefore, $W F$ ' is also confusion-free well-formed.

The theorem is proved.

Theorem 3: Given a confusion-free well-formed workflow $W F=\left(T, P, C, A, S_{0}\right)$, deleting a task $T_{k} \in T$ and all precedence arcs starting from or ending up to $T_{k}$, the obtained new workflow is denoted by $W F^{\prime}=\left(T^{\prime}, P^{\prime}, C^{\prime}, A^{\prime}, S_{0}{ }^{\prime}\right)$. If the deletion of does not cause an unhealthy loop, then $W F^{\prime}$ is also a confusion-free well-formed workflow.

Proof: Since the deletion does not cause an unhealthy loop, the workflow is guaranteed to finish. We only need to prove all tasks in the new workflow are executable, Consider the following four cases:

Case 1): $* T_{k}=T_{k} *=\varnothing$.

In this case, $T_{k}$ is an isolated task, so its presence or absence has no impact on the execution of the rest of the workflow.

Case 2): $* T_{k}=\varnothing, T_{k} * \neq \varnothing$.

In this case, $T_{k}$ has no predecessors, so we only need to prove that all tasks that are immediate successors of $T_{k}$ are still executable after removing in $T_{k}$.

Suppose $T_{i} \in T_{k}{ }^{*}$. If $T_{i}$ is an AND-in task in $W F$, then that $W F$ is well-formed implies that $T_{i}$ is executable, i.e. there is a state $S_{a}$ in $W F$ such that all tasks in $* T_{i}$ have state value 2. If $\forall T_{j} \in * T_{i} \backslash\left\{T_{k}\right\}, T_{j}$ is not a (either immediate or not) successor of $T_{k}$, then $T_{j}$ is executable in $W F^{\prime}$, then there must be a corresponding state $S_{a}$ ' in $W F^{\prime}$ such that $S_{a}{ }^{\prime}=S_{a} \backslash\left\{S_{a}{ }^{\prime}\left(T_{i}\right)=2\right\}$, which means $T_{i}$ is still executable in $W F^{\prime}$. If $\exists T_{j} \in$ $* T_{i} \backslash\left\{T_{k}\right\}, T_{j}$ is a successor of $T_{k}$, then we can always find a task $T_{l}$ such that $T_{l} \in T_{k}{ }^{*}$ and $T_{j}$ is a successor of $T_{l}$. Without loss of generality, we assume that there is no other task that is also an immediate successor of $T_{k}$ and sits in between $T_{l}$ and $T_{j}$. We have already proved that $T_{l}$ is executable after removing $T_{k}$. So $T_{j}$ is also executable after removing $T_{k}$, which further indicates that $T_{i}$ is executable in $W F$ '.

Now we consider the case that $T_{i}$ is an XOR-in task in $W F$. Again, $W F$ is wellformed implies that $T_{i}$ is executable, and it also implies that all of its $\mathrm{s}$ are executable. So, removing $T_{k}$ has no impact to the executability of $T_{i}$. In other words, $T_{i}$ is still executable in $W F$ '. 
Case 3) $* T_{k} \neq \varnothing, T_{k}^{*}=\varnothing$.

In this case, $T_{k}$ has no successors, so removing $T_{k}$ has no impact to the executability of $T_{i}$. Since $W F$ is well-formed, all tasks in $T \backslash\left\{T_{k}\right\}$ are executable in $W F$. Thus all tasks in $W F$ ' are also executable. Moreover, if $S_{q}$ is an ending state of $\mathrm{WF}$, then $S_{q}{ }_{q}=S_{q} \backslash$ $\left\{S\left(T_{k}\right)=2\right\}$ is an ending state of $W F^{\prime}$. Therefore, $W F^{\prime}$ is confusion-free well-formed. Also, it is obvious that the two structural properties of confusion-free workflow are not affected by removing $T_{k}$. So $W F$ ' is also confusion free.

Case 4) $* T_{k} \neq \varnothing, T_{k}^{*} \neq \varnothing$.

This case can be viewed as a combination of Case 2 and Case 3. We first split $T_{k}$ into $T_{k 1}$ and $T_{k 2}$, where $T_{k 1}$ and $T_{k 2}$ are connected as shown in $W F_{B}$ of Fig. 2, $T_{k l}$ satisfies condition 3) and $T_{k 2}$ satisfies condition 2). According to Lemma 1 we know the obtained workflow is well-formed and confusion free. Then we remove the precedence constraint between $T_{k 1}$ and $T_{k 2}$, and the obtained workflow, like the $W F_{A}$ of Fig. 2, is also well-formed and confusion free according to Theorem 2. Finally, applying the proof for Case 2) and Case 3) results in that the obtained workflow after removing $T_{k l}$ and $T_{k 2}$, which is $W F^{\prime}$ stated in this theorem, is well-formed and confusion free.

The theorem is proved.

Theorem 3 shows a distinguishing feature of confusion-free workflows. That is, when we delete any task and its associated precedence arcs from a workflow, as long as it does not cause any unhealthy loop, the remaining workflow is still well-formed and confusion-free. However, it should be pointed out that, if the task to be deleted is not an isolated task, then deleting it will cause changes to the precedence relations among the remaining tasks. So in most cases, after we delete a task, we need to adjust the precedence relations among those tasks that are s or immediate successors.

When adding a precedence arc between two tasks, say $T_{i}$ and $T_{j}$, the relationship between these two tasks in the original workflow has an impact to the wellformedness of the resultant workflow. More specifically, if $T_{j}$ is not a predecessor of $T_{i}$, then setting $p_{i j}=0$ will not cause a loop. Otherwise, a loop will be introduced. In the latter case, if $T_{i}$ is an "AND-in" task, then $T_{i}$ will never be executable because of the existence of deadlock: $T_{i}$ is not executable until $T_{j}$ is executed; meanwhile $T_{j}$ is not executable until $T_{i}$ is executed. In addition, as a general rule, one should not introduce a precedence arc between two exclusive tasks, because it violates logic consistency. The following theorem addresses adding precedence constraint to a workflow. Due to space limitation, we only give proof sketch to this theorem.

Theorem 4: Let $W F=\left(T, P, C, A, S_{0}\right)$ be a well-formed confusion free workflow with $T_{i}, T_{j} \in T$ where $p_{i j}=0$ and $T_{i}$ and $T_{j}$ are not mutual exclusive. $W F$ ' is obtained by adding a precedence arc between $T_{i}$ and $T_{j}$, i.e. setting $p_{i j}$ to 1 . Then $W F^{\prime}$ is confusionfree well-formed if it matches one of the following cases:

1) $T_{i}$ is of the same type in both $W F$ and $W F$, so is $T_{j}$.

2) If the addition introduces a loop, then the loop must be healthy.

Proof sketch: Condition 1) ensures confusion free and task executability. Condition 2) guarantees the new workflow to finish.

The importance of the above theorems is that they allow a progressive workflow wellformedness verification, which significantly reduces the complexity of large-scale 
model analysis. This is particularly useful in modeling and enacting incident command systems where timing is so critical to mission success. In the next section we will show how to apply these theorems.

\section{Example}

We illustrate the use of the five theorems through changes to the workflow model shown in Fig. 1.

Suppose that the model is incomplete. Now we add task $T_{8}$ to the model such that $T_{8}$ is the immediate successor of $T_{7}$ and it has no successors. The new model is shown in Fig. 4. Based on Theorem 1, Case 3, the workflow of Fig. 4 is well-formed and confusion-free.

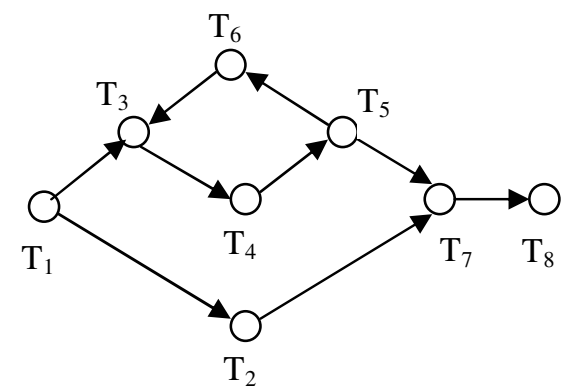

Fig. 4. Add task $T_{8}$

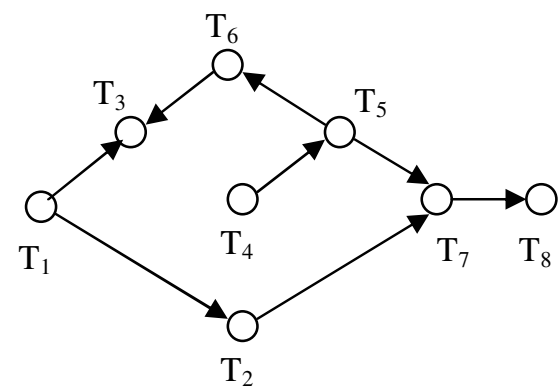

Fig. 6. Delete precedence arc between $T_{3}$ and $T_{4}$

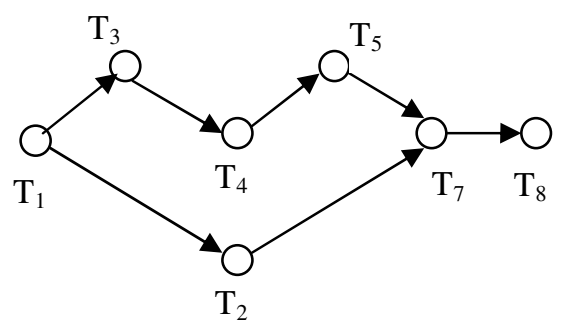

Fig. 5. Delete task $T_{6}$

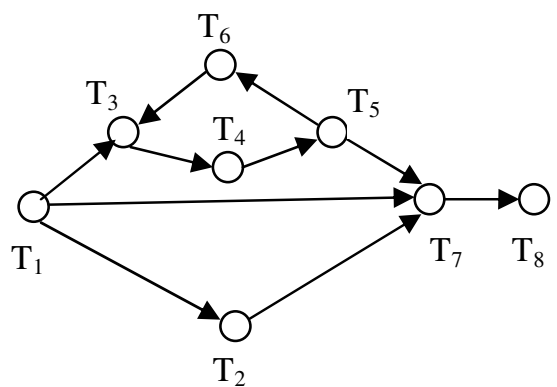

Fig. 7. Add precedence arc between $T_{1}$ and $T_{7}$

The workflow in Fig. 5 is obtained by deleting task $T_{6}$ from Fig. 4. Because the workflow of Fig. 4 is well-formed and confusion-free, based on Theorem 2, the workflow of Fig. 5 is also well-formed and confusion-free. Note that deleting $T_{7}$ from Fig. 4 is not allowed, because it will make the loop of $T_{3}, T_{4}, T_{5}$ and $T_{6}$ unhealthy - no exit.

Fig. 6 shows a case where a precedence arc between two tasks that are in a loop is deleted. After deleting the arc between $T_{3}$ and $T_{4}, T_{4}$ becomes unconditionally 
executable. It is not hard to find that there is no dangling task in this model, and the workflow, depending on $T_{6}$ or $T_{7}$ is chosen to execute, will either end at state $(2,2,2$, $2,2,2,0,0)$ or state $(2,2,2,2,2,0,2,2)$, respectively. So the workflow is wellformed and confusion-free. This conclusion can be drawn immediately by applying Theorem 3, because $T_{3}$ is a XOR-in task.

In Fig. 7, a precedence arc between $T_{1}$ and $T_{7}$ is added, and it is important that $T_{1}$ remain as an AND-in task and $T_{7}$ remain as an AND-in task. Then based on Theorem 4, the workflow is well-formed and confusion-free.

Note that a change to a workflow may take several atomic steps, and the wellformedness may be violated before the change is complete. We allow this to happen as long as the model after the change is still well-formed.

\section{Tool Support}

We have implemented a prototype that supports the WIFA approach [17]. It has been applied to an incident command system exercise for training purposes.

The central tool component is the model constructor, which handles the visual displays, UI functionality, organization of objects in a workflow, and access to the validation and simulation functions (see Fig. 8). The workflow editor has a wide range of drag-and-drop features that allow users to easily build workflows following an intuitive process. Pre-built workflow components can be used to speed up the editing process. As soon as the workflows are built they can be validated using the validator component of the tool, in order to assess that the workflow follows the well-formed workflow definitions presented earlier. In addition, the validation process produces two separate outputs. One output is the validation report which includes errors in the workflow design and suggestions on how to fix them. The second output is the workflow reachable states that can be analyzed later.

Once the workflows are validated they can be executed using the simulator component. The simulation can be done either manually or by specifying a time interval. Throughout the simulation the user has the option of stepping back, pausing, and stopping. During these simulations the users can still adjust their workflows, by interactively making modifications and validating them, as it was described earlier in the paper. All workflows are stored as XML files.

Since intuitiveness is of paramount importance for this tool, a usability assessment was done on the workflow editor at Monmouth University in order to assess both users' perceptions of the tool and their behavior while actually creating workflows. Fifteen participants underwent an approximately 45-minute testing session that consisted of three phases. Participants ranged from undergraduate sophomores with no programming expertise to more experienced software engineering graduate students. Afterwards, participants were asked to complete a questionnaire. The results of the questionnaire suggest that, in general, the workflow editor was considered useful and that its usability was acceptable. The only aspect that was considered less intuitive was the four types o tasks that we defined in WIFA. In order to avoid this drawback, we have since incorporated into the tool the capability of using abstract task types that don't require the users to specify the task types. Other minor findings of the usability study are currently being included in the prototype. 


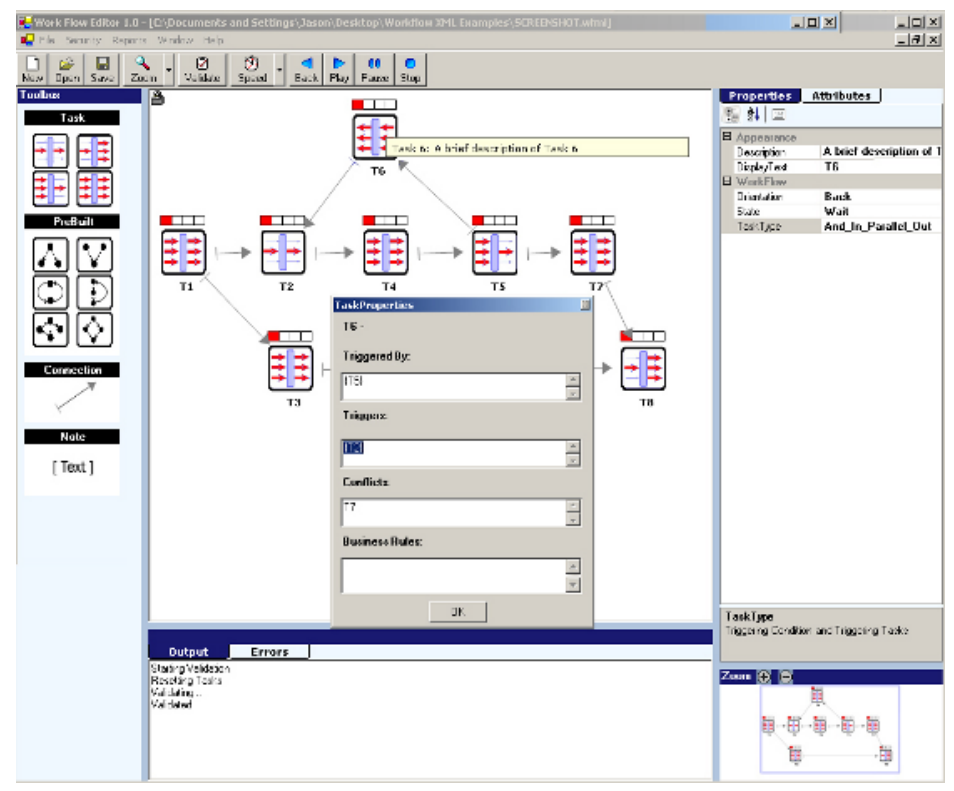

Fig. 8. WIFA prototype

\section{Concluding Remarks}

In this paper we explored the dynamic properties of the WIFA workflow engine. More specifically, we developed a set of theorems to guide the dynamic modeling, modification and verification of well-formed workflows. The importance of this work relies in these theorems that allow a progressive workflow well-formedness verification, which significantly reduces the complexity of large-scale model analysis. This is particularly useful in modeling and enacting incident command systems where timing is so critical to mission success.

We are also working on extending WIFA with the data dimension, and with capabilities for inter-organizational workflow modeling and analysis, to be able to represent the interactions between different people and organizations that need to work together for achieving different business goals.

\section{References}

1. W.M.P. van der Aalst, "Verification of Workflow Nets", Proceedings of Application and Theory of Petri Nets, LNCS, Volume 1248, pp. 407-426, 1997.

2. W.M.P. van der Aalst, "Three Good Reasons for Using a Petri Net-Based Workflow Management System”, Proceedings of the International Working Conference on Information and Process Integration in Enterprises (IPIC'96), pp. 179-201, Nov 1996.

3. W.M.P. van der Aalst, A.H.M. ter Hofstede, and M. Weske, "Business Process Management: A Survey." International Conference on Business Process Management (BPM 2003), LNCS, volume 2678, pages 1-12. Springer-Verlag, Berlin, 2003. 
4. W.M.P. van der Aalst, A.H.M. ter Hofstede, B. Kiepuszewski, and A.P. Barros, "Workflow Patterns," Eindhoven University of Technology, Eindhoven, 2000.

5. N. R. Adam, V. Atluri and W. Huang, "Modeling and Analysis of Workflows Using Petri Nets", Journal of Intelligent Information Systems, pp. 131-158, March 1998.

6. Agostini A. and G. DeMichelis, "A light workflow management system using simple process models", International Journal of Collaborative Computing (16), 2000, pp. 335-363.

7. P. C. Attie, M. P. Singh, A. Sheth and M. Rusibkiewicz, "Specifying Interdatabase Dependencies," Proc. 19th International Conf. on Very Large Database, pp.134-145, 1993.

8. F. Casati, S. Ceri, B. Pernici, G. Pozzi, "Workflow evolution”, Data and Knowledge Engineering Journal, Elsevier, vol. 24 (3), 1998, pp. 211-238.

9. P. Dourish, "Process Descriptions as Organizational Accounting Devices: The Dual use of Workflow Technologies", Paper presented at GROUP'01, (ACM), Sept. 30-Oct. 3, 2001, Boulder, Colorado, USA.

10. C. Ellis, K. Keddara, "A workflow Change is a workflow", Proceedings BPM'00, LNCS, vol. 1806, 2000, pp. 516-534.

11. M. Kradolfer, A. Geppert, "Dynamic workflow schema evolution based on workflow type versioning and workflow migration”, Proceedings of CoopIS'99, Edinburgh, 1999, pp. 104-114.

12. P. Lawrence, editor, "Workflow Handbook 1997, Workflow Management Coalition", John Wiley and Sons, New York, 1997.

13. D.C. Marinescu, Internet-Based Workflow Management: Towards a Semantic Web, Wiley Series on Parallel and Distributed Computing, vol. 40, Wiley-Interscience, NY, 2002.

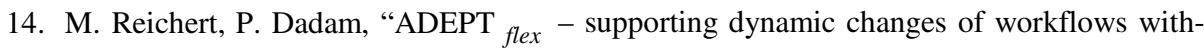
out losing control”, Journal of Intelligent Information Systems, 10 (2), 1998, pp.93-129.

15. D. Rosca, S. Greenspan, C. Wild, "Enterprise Modeling and Decision-Support for Automating the Business Rules Lifecycle", Automated Software Engineering Journal, Kluwer Academic Publishers, vol.9, pp.361-404, 2002.

16. M.P. Singh, G. Meredith, C. Tomlinson, and P.C. Attie, "An Event Algebra for Specifying and Scheduling Workflows," Proceedings 4th International Conference on Database System for Advance Application, pp. 53-60, 1995.

17. M. Stoute, J. Wang, and D. Rosca, "Workflow Management Tool Support for an Incident Command System", accepted for publication in the Proceedings of ICNSC'06, Miami, FL, 2006

18. J. Wang, Timed Petri Nets: Theory and Application, Kluwer Academic Publishers, 1998, ISBN: 0-7923-8270-6.

19. J. Wang, D. Rosca, W. Tepfenhart, A. Milewski and M. Stoute, “An Intuitive Formal Approach to Dynamic Workflow Modeling and Analysis," Proceedings of the $3^{\text {rd }}$ Conference on Business Process Management, Nancy, France, Sept. 6-8, 2005.

20. M. Weske, "Formal Foundation and Conceptual Design of dynamic adaptations in a workflow management system", Proceedings of HICSS-34, 2001. 